\title{
Time- and State-Dependent Pricing: A Unified Framework*
}

\author{
Marco Bonomo \\ Graduate School of Economics, \\ Getulio Vargas Foundation
}

\author{
Carlos Carvalho \\ PUC-Rio
}

\author{
René Garcia \\ EDHEC Business School
}

December 2012

\begin{abstract}
We develop a tractable unified framework for solving optimal time- and state-dependent price-setting problems. We illustrate our approach by solving a price-setting problem where adjustments are costly, and there are two types of information. One type of information is freely available and flows continuously, while the other type is costly and requires the payment of a lump-sum cost to be obtained. Our choice of state variables is key to make the problem tractable. Specifically, we replace the usual state variable in state-dependent pricing problems the discrepancy between the firm's price and its frictionless optimal level - with its expectation conditional on the firm's information set, and augment the state space with the time elapsed since the last date when information was fully factored into the pricing decision ("information date"). This allows us to formulate the price-setting problem as two-dimensional optimal stopping problems. Our analysis uncovers new insights about price setting. Time dependency in pricing rules arises as a consequence of the build up of unobserved information. In these circumstances, the inaction region changes as a function of the time elapsed since the last information date. When the next information date is known, the presence of menu costs produces an extreme form of inaction: irrespective of the size of the expected price discrepancy, it is never optimal to adjust just prior to the information date.
\end{abstract}

JEL classification codes: E00, E31

Keywords: menu costs, information costs, infrequent information, sticky information, inattentiveness, optimal price setting, state-dependent pricing, time-dependent pricing

*An earlier working paper version circulated under the title "State-Dependent Pricing under Infrequent Information: A Unified Framework". For comments and suggestions we thank Mike Golosov, Virgiliu Midrigan, and seminar participants at ESEM 2011, Advances in Macroeconomics Workshop (FGV-Rio), ESWM 2011, SBE 2010, LACEA 2010, INSPER-SP. Emails: marco.bonomo@fgv.br, cvianac@econ.puc-rio.br, rene.garcia@edhec.edu. 


\section{Introduction}

The recent availability of vast amounts of micro price data has generated renewed interest in price setting among macroeconomists, especially since the seminal work of Bils and Klenow (2004). This has lead to noteworthy developments in this field - in particular in terms of microfounded models with explicit price-setting frictions. Many papers have expanded the frontier of so-called menu-cost models. ${ }^{1}$ In addition, recent work has analyzed the implications of explicit informational frictions for price setting behavior. ${ }^{2}$ Despite this progress, the price setting literature still appears to be guided by a dichotomy between time-dependent and state-dependent pricing rules, ${ }^{3}$ and these two branches have developed essentially in paralell.

In this paper we develop a tractable unified framework for solving optimal time- and statedependent price-setting problems. Although our framework may be used to study several models with adjustment costs and infrequent information, including most price-setting problems analyzed previously in the literature, ${ }^{4}$ here we focus on a specific model where the use our approach seems essential. It is a model with costly price adjustments where part of the relevant information flows continuously and can be factored into pricing decisions somewhat costlessly, while the other part can only be incorporated by paying a lump-sum information cost $(F) .{ }^{5}$ So, the model incorporates both costly and free information in a otherwise standard menu-cost model. ${ }^{6}$

The key to making our approach tractable is our choice of state variables. We rely on a commonly used second-order approximation to the profit loss due to price-setting frictions, which implies that these losses are proportional to the square of the discrepancy between the firm's (log) price and its frictionless optimal level (henceforth the price discrepancy). Because of the intertemporal nature of the problem, the price setter must forecast the path of these squared discrepancies. Given the stochastic processes used to model the frictionless optimal price, these forecasts turn out to be a very simple function of two conveniently chosen state variables: the conditional expectation of the price discrepancy and the time elapsed since the last date when

\footnotetext{
${ }^{1}$ Some examples are Golosov and Lucas (2007), Gertler and Leahy (2008), Midrigan (2011), and Nakamura and Steinsson (2010).

${ }^{2}$ For instance, Reis (2006), Woodford (2009), Maćkowiak and Wiederholt (2009).

${ }^{3}$ The titles of the following papers, among others, are illustrative of this dichotomy: "Time- or state-dependent price setting rules? Evidence from micro data," (Dias, Marques and Silva 2007), "State-Dependent or TimeDependent Pricing: Does It Matter for Recent U.S. Inflation?" (Klenow and Kryvtsov 2008), "Is Firm Pricing State or Time Dependent? Evidence from U.S. Manufacturing" (Midrigan 2010).

${ }^{4}$ In Bonomo, Carvalho, Garcia (2011) we use this framework to solve several models with costly adjustment and/or infrequent information.

${ }^{5}$ Gorodnichenko (2008), Knotek (2009) and Klenow and Willis (2007) propose menu-cost models in which firms continuously incorporate partial information into pricing decisions.

${ }^{6}$ In general information frictions of one type has been incorporated into price-setting models (e.g.Caballero 1989, Reis 2006, Moscarini 2004, Bonomo and Carvalho 2004,2010, Woodford 2009, Gorodnichenko 2008, Knotek 2009, Klenow and Willis 2007, and Alvarez, Lippi, and Paciello 2011).
} 
information was fully factored into the pricing decision (henceforth information dates). This choice of state variables allows us to cast each pricing problem as a two-dimensional optimal stopping problem. For each case that we analyze, we rewrite the Bellman equation that characterizes the firm's value function in the inaction region as either an ordinary differential equation in one of those two state variables, or as a partial differential equation in both variables. Boundary conditions dictated by the nature of the problem pin down the solution, which in some cases can be written in (almost) closed form, or is otherwise obtained numerically through algorithms that make use of finite-difference methods.

Our unified framework makes it clear that pure time- and pure state-dependent pricing policies are special cases of more general time-and-state-dependent rules that turn out to be optimal in the presence of both adjustment and information frictions. Time dependency arises as a consequence of the build up of unobserved information. In these circumstances, the optimal policy is characterized by an inaction region that varies with the time elapsed since the last information date. The reason is that the option value of waiting for new information tends to increase with time, due to the accumulation of underlying (unobserved) innovations. To the best of our knowledge, this dependence of the inaction region on the age of the firm's information set is a feature of optimal pricing policies that had not emerged in previous work.

When the next information date is known, the presence of menu costs produces an extreme form of inaction: irrespective of the size of the expected price discrepancy, it is never optimal to adjust just prior to the information date. Notice that this is true even if the partial information available indicates that the firm's price is very far from the frictionless optimal one. Finally, our results show that despite the presence of menu costs, firms may choose to change prices with only partial information.

Section 2 introduces our framework, with a focus on our choice of state variables. Then, in Section 3 we introduce the partial information model. In this costly adjustment model, there is always some continuous flow of information that can be factored into pricing decisions somewhat costlessly, and some information that can only be incorporated by paying a lump-sum information cost $(F) .{ }^{7}$ A firm always has the option to incur the lump-sum adjustment cost $(K)$ and make a price adjustment based on its current information about the underlying frictionless optimal price. It can also incur the lump-sum information $\operatorname{cost} F$ to become fully informed about that frictionless price. the optimal pricing rule features both infrequent price changes and infrequent incorporation of information into prices. It is characterized by an inaction region for price adjustment and information gathering/processing, which is defined by the intersection between an adjustment inaction

\footnotetext{
${ }^{7}$ Gorodnichenko (2008), Knotek (2009) and Klenow and Willis (2007) propose menu-cost models in which firms continuously incorporate partial information into pricing decisions.
} 
region and an information inaction region. The borders inherited from the adjustment inaction region trigger uninformed adjustments, while the border inherited from the information inaction region triggers information gathering/processing. It is never optimal to make a partially informed price adjustment just prior to an information date. Rather than incurring the menu cost to make such an adjustment and then immediately incurring the information gathering/processing cost, it is always better to reverse the order of these actions and keep the option to adjust, to be exercized or not depending on the new information. We also study a simpler variant where besides the continuous flow of information there is an exogenous arrival of information at deterministic time. This case illustrates more clearly the principle that is always worth waiting for information arrival, instead of adjusting immediately, when the arrival is imminent.

In Section 4 we analyze a particular case of the previous model where there is no freely available partial information. Then the model becomes one in which all the information is costly. The firm's problem in this context is to choose adjustment and information dates subject to lump-sum information and adjustment costs. Gorodnichenko (2008), Abel, Eberly, and Panageas (2010), Alvarez, Guiso, and Lippi (2010), and Alvarez, Lippi, and Paciello (2011) also analyze models in which agents face information and adjustment costs. The main difference between our work and theirs is that our approach to solving the problem with information and adjustment costs encompasses cases in which uninformed adjustments are optimal. ${ }^{8}$

\section{The framework}

We start by setting up the problem of a firm that faces a menu cost to change its price, and is subject to information frictions that require it to pay a lump-sum cost in order to fully gather and process information about the relevant underlying state of the economy. Our formulation encompasses cases in which the firm has continuous partial information about such state.

The general idea behind the price-setting problems that we study is that, in the absence of frictions - and thus under full information - a firm would set its price $p_{t}$ equal to the so-called

\footnotetext{
${ }^{8}$ In Gorodnichenko's (2008) model, firms always have some (imperfect) information about the frictionless optimal price. Abel, Eberly and Panageas (2010) show that their asymptotic result of convergence to a purely time-dependent portfolio management policy survives if one allows for what they refer to as "automatic transfers" between the agent's investment portfolio and the transactions account. However, they do not investigate the optimality of automatic transfer plans. Alvarez, Lippi and Paciello (2010, section 7.3) and Alvarez, Guiso and Lippi (2010, Appendix AA-3) discuss the case of adjustment without information. They provide sufficient conditions under which such uninformed adjustments are not optimal. For the price-setting problem, Alvarez, Lippi and Paciello (2010) show that this is the case for a sufficiently small rate of inflation. For the problem of asset management with consumption of durables, Alvarez, Guiso and Lippi (2010) show that this is the case when there is no uncertainty in asset returns. These two papers then focus on parameterizations that satisfy those sufficient conditions, and otherwise prevent agents from making uninformed adjustments by imposing the restriction that adjustment requires observation. For a more extensive discussion of the differences between our work and these papers, see Bonomo, Carvalho, and Garcia (2011).
} 
frictionless optimal price $p_{t}^{*}$ - which is its instantaneously profit-maximizing price. ${ }^{9}$ In the presence of impediments to such "ideal" price setting, firms choose the optimal pricing policy subject to lumpsum adjustment cost $K$ and information cost $F$ in order to maximize intertemporal profits. This policy consists of a sequence of information dates $\left\{t_{j}^{0}\right\}_{j=1}^{\infty}$, and for each information date indexed $j\left(t_{j}^{0}\right)$ a subsequent sequence of pairs of price adjustment dates and new prices $\left\{t_{j}^{n}, p_{t_{j}^{n}}\right\}_{n=1}^{N_{j}}$ chosen between this $\left(t_{j}^{0}\right)$ and the next information date $\left(t_{j+1}^{0}\right) \cdot{ }^{10}$ Any deviation between a firm's actual price, $p_{t}$, and $p_{t}^{*}$ - what we refer to as a price discrepancy - entails an instantaneous flow "cost" in the form of foregone profits. As we show in Appendix B, these "discrepancy costs" can be taken as being approximately equal to the square of the price discrepancy: $\left(p_{t}-p_{t}^{*}\right)^{2}$. The objective of firms is to minimize the present discounted value of expected total costs, which comprise the (integral of) flow discrepancy costs plus discounted adjustment and information costs.

Formally, the firm's problem at any information date labeled $t_{0}^{0}$ is given by the following intertemporal optimization program: ${ }^{11}$

$$
\begin{gathered}
V\left(s_{t_{0}^{0}}, p_{t_{-1} N_{-1}}\right)= \\
\left\{t_{j}^{0},\left\{t_{j}^{n}, p_{t_{j}^{n}}\right\}_{n=1}^{N_{j}}\right\}_{j=1}^{\infty} E_{t_{0}^{0}} \sum_{j=0}^{\infty} E_{t_{j}^{0}}\left\{\begin{array}{c}
e^{-\rho\left(t_{j+1}^{0}-t_{0}^{0}\right)} F+\int_{t_{j}^{0}}^{t_{j}^{1}} e^{-\rho\left(r-t_{0}^{0}\right)}\left(p_{t_{j-1}^{N_{j-1}}}-p_{r}^{*}\right)^{2} d r \\
+\sum_{n=1}^{N_{j}-1}\left[\int_{t_{j}^{n}}^{t_{j}^{n+1}} e^{-\rho\left(r-t_{0}^{0}\right)}\left(p_{t_{j}^{n}}-p_{r}^{*}\right)^{2} d r+e^{-\rho\left(t_{j}^{n}-t_{0}^{0}\right)} K\right. \\
+\int_{t_{j}^{N_{j}}}^{0} e^{-\rho\left(r-t_{0}^{0}\right)}\left(p_{t_{j}^{N_{j}}-p_{r}^{*}}\right)^{2} d r+e^{-\rho\left(t_{j}^{N_{j}}-t_{0}^{0}\right)} K
\end{array}\right\},
\end{gathered}
$$

where $s_{t_{0}^{0}}$ is the initial state, $p_{t_{-1}^{N_{-1}}}$ the inherited price, $\rho$ is the time discount rate, and $E_{t}$ denotes the expectation operator conditional on time $t$ information.

Under infrequent information about $p_{t}^{*}$, in order to evaluate the expected flow cost due to price discrepancies the firm must form a probabilistic assessment of $p_{t}^{*}$ given its information. We can decompose the instantaneous expected flow cost due to a price discrepancy at time $t$ as:

$$
\begin{aligned}
E_{t}\left(p_{t}-p_{t}^{*}\right)^{2} & =\left(p_{t}-E_{t} p_{t}^{*}\right)^{2}+E_{t}\left(p_{t}^{*}-E_{t} p_{t}^{*}\right)^{2} \\
& =\left(p_{t}-E_{t} p_{t}^{*}\right)^{2}+\operatorname{Var}_{t}\left(p_{t}^{*}\right)
\end{aligned}
$$

\footnotetext{
${ }^{9}$ In Appendix A we present a simple general equilibrium model that yields an expression for the (logarithm of the) frictionless optimal price for a firm, $p_{t}^{*}$, as the sum of two components - an aggregate (nominal aggregate demand) and an idiosyncratic (productivity) component.

${ }^{10}$ Among the adjustment date/price pairs we allow for a sequence before the first information date $\left\{t_{0}^{n}, p_{t_{0}^{n}}\right\}_{n=1}^{N_{j}}$.

${ }^{11}$ The formalization of the problem starting at an arbitrary date is similar, but heavier on notation.
} 
$\operatorname{Var}_{t}$ denotes the conditional variance given time $t$ information. The first term in the right-hand side of (2) represents the flow cost of deviating from the expected level of the frictionless optimal price, and the second term represents the expected flow cost from not continuously entertaining full information about the latter. In the absence of adjustment costs, $p_{t}$ would be set equal to $E_{t} p_{t}^{*}$, reducing the first part of the discrepancy cost to zero. Otherwise the firm must optimally solve the trade-off between letting $p_{t}$ drift away from $E_{t} p_{t}^{*}$, and paying the cost to adjust.

As for the second term in (2), it is zero when information can be fully and continuously incorporated into the pricing decision at no cost, as in standard menu-cost models. If information gathering and processing is costly, the firm can reduce the second term at the expense of incurring the information cost. If the firm has continuous access to partial information about $p_{t}^{*}$, the conditional variance $\operatorname{Var}_{t}\left(p_{t}^{*}\right)$ refers to the component of the frictionless optimal price that is only observed at a cost.

We assume throughout that $p_{t}^{*}$ follows a Markovian stochastic process, and that for any $\Delta t>0$ the distribution of $p_{t+\Delta t}^{*}-p_{t}^{*}$ depends only on $\Delta t$. In particular, this implies that the conditional variance $\operatorname{Var}_{t}\left(p_{t}^{*}\right)$ depends only on the time elapsed since the last information date. From this assumption and the structure of the firm's problem, given an information date $t_{0}$, the value function at a time $t>t_{0}$ - the optimized value of the firm's dynamic cost-minimization problem defined in (1) - can be written in terms of two state variables: the time elapsed since the last information date, denoted by $\tau \equiv t-t_{0}$, and the deviation of $p_{t}$ from its expected frictionless optimal level given its current information (which we refer to as the expected discrepancy), defined as:

$$
z_{t} \equiv p_{t}-E_{t} p_{t}^{*}
$$

We can thus write the firm's expected discrepancy cost as a function of $\tau$ and $z$ :

$$
\begin{aligned}
E_{t}\left(p_{t}-p_{t}^{*}\right)^{2} & =z_{t}^{2}+\operatorname{Var}_{t}\left(p_{t}^{*}\right) \\
& \equiv f\left(z_{t}, \tau\right)
\end{aligned}
$$

With lump-sum adjustment and information costs, price changes and information gathering will be infrequent. In the inaction region (i.e., absent any price change and/or information gathering), the value function $V$ obeys the following Bellman equation:

$$
V\left(z_{t}, \tau\right)=f\left(z_{t}, \tau\right) d t+e^{-\rho d t} E_{t} V\left(z_{t+d t}, \tau+d t\right)
$$

Equation (5) is valid for a variety of environments, including the standard full-information case. ${ }^{12}$

\footnotetext{
${ }^{12}$ In an earlier working paper version (Bonomo, Carvalho and Garcia 2010) we use this framework to study a
} 
In the next section we apply this framework to a new price-setting problem.

\section{A model with menu costs and costly partial information}

We develop a price-setting model with costly price adjustments in which there is always some continuous flow of information that can be factored into pricing decisions somewhat costlessly, and some information that can only be incorporated by paying a lump-sum information cost $(F) .{ }^{13} \mathrm{~A}$ firm always has the option to incur the lump-sum adjustment cost $(K)$ and make a price adjustment based on its current information about the underlying frictionless optimal price. It can also incur the lump-sum information cost $F$ to become fully informed about that frictionless price. These two possible actions imply an optimal inaction region, which we describe heuristically before showing how we solve this pricing problem.

Upon incurring the lump-sum adjustment cost $K$, a firm can choose a new price, and will do so in order to set the expected price discrepancy $z_{t}$ to an optimal level $c_{t}$. For a given information set, adjustment is only worthwhile if the expected price discrepancy is "large enough" to justify incurring the menu cost. This implies that at each point in time there are bounds $l_{t}$ and $u_{t}$ on the expected discrepancy such that the firm will increase its price whenever $z_{t}$ is less than $l_{t}$, and decrease its price whenever $z_{t}$ exceeds than $u_{t}$. The assumptions about the stochastic process for $p_{t}^{*}$ that we specify subsequently imply that the policy functions $\left\{l_{t}, c_{t}, u_{t}\right\}$ do not depend on calendar time per se, but only on the time elapsed since the last information date $(\tau)$. We thus write $\{l(\tau), c(\tau), u(\tau)\}$, and refer to $\{l(\tau), u(\tau)\}$ as the bounds of the adjustment inaction region.

Turning to the information decision, upon incurring the information cost $F$ the firm learns the history of innovations to its frictionless optimal price that had not been freely observed. This amounts to a shock to the expectation of the price discrepancy that the firm held just prior to gathering information. Because of the lump-sum nature of the information cost, it is clear that the firm will not gather information continuously. At any given time $t$, gathering information is only worthwhile if the cost of not observing the innovations that have occurred since the last information date exceeds the information cost $F$. As is clear from (4), as long as the conditional variance $\operatorname{Var}_{t}\left(p_{t}^{*}\right)$ increases with the time elapsed since the last information date, the cost of not observing the underlying innovations will increase over time. The assumptions about $p_{t}^{*}$ that we specify subsequently ensure that the cost of not observing the underlying innovations will eventually exceed the information cost for any given expected discrepancy. This implies, for each expected price

variety of price setting problems with explicit adjustment and/or information frictions, including the most common specifications found in the literature, as well as some novel problems.

${ }^{13}$ Gorodnichenko (2008), Knotek (2009) and Klenow and Willis (2007) propose menu-cost models in which firms continuously incorporate partial information into pricing decisions. 
discrepancy $z$, a time elapsed since the last information date $\left(\tau^{*}(z)\right)$ that will trigger information gathering. We refer to $\tau^{*}(z)$ as the boundary of the information inaction region.

\subsection{Solution}

We now characterize the conditions that determine the optimal pricing rule and solve the problem numerically. We first characterize the partial differential equation which governs the evolution of the value function in the inaction range. We then specify the boundary and optimality conditions which characterize the optimal pricing policy.

\subsubsection{The value function PDE}

We assume that the frictionless optimal price process is given by: ${ }^{14}$

$$
d p_{t}^{*}=\mu d t-\sigma_{i} d W_{i, t}-\sigma_{a} d W_{a, t}
$$

where $W_{i, t}$ and $W_{a, t}$ are independent standard Wiener processes. Information about $W_{i, t}$ is continuously and freely available, and costless to process. In contrast, gathering and processing information about $W_{a, t}$ is costly.

Between information dates and in the absence of price adjustments (i.e., in the inaction region), $z_{t}$ changes continuously because of both the drift $\mu$ and the $W_{i, t}$ process:

$$
d z_{t}=-\mu d t+\sigma_{i} d W_{i, t}
$$

$W_{a, t^{-}}$driven uncertainty also impacts the expected discrepancy costs due to the build up of unobserved innovations. Thus, the instantaneous flow-cost function is given by:

$$
f\left(z_{t}, \tau\right)=z_{t}^{2}+\operatorname{Var}_{t}\left(p_{t}^{*}\right)=z_{t}^{2}+\sigma_{a}^{2} \tau
$$

where, again, $\tau$ denotes the time elapsed since the last information date. Taking into account the process for $z(6)$ and the flow cost (7) in the differential form of the Bellman equation (5), and applying Ito's lemma, results in the following partial differential equation for the value function $V$ :

$$
\frac{1}{2} \sigma_{i}^{2} V_{z z}(z, \tau)-V_{z}(z, \tau) \mu+V_{\tau}(z, \tau)-\rho V(z, \tau)+z^{2}+\sigma_{a}^{2} \tau=0
$$

\footnotetext{
${ }^{14}$ As mentioned previously, the two-component representation of $p_{i t}^{*}$ can be justified from first principles, as in Appendix A.
} 


\subsubsection{The adjustment decision}

Because adjustment costs are lump-sum, any adjustment is made to the point that minimizes the value function. So the target point $c(\tau)$ when the time elapsed is $\tau$ satisfies:

$$
c(\tau)=\arg \min _{z} V(z, \tau) .
$$

Since firms always have the option to incur the adjustment cost $K$ and reset the expected discrepancy to $c(\tau)$, the value function must always satisfy:

$$
V(z, \tau) \leq V(c(\tau), \tau)+K
$$

The bounds that define the adjustment inaction region, $l(\tau), u(\tau)$, are functions of the time elapsed since the last information date that imply indifference between adjusting and not adjusting. They satisfy the value-matching conditions that obtain when (10) holds with equality:

$$
\begin{aligned}
V(l(\tau), \tau) & =V(c(\tau), \tau)+K, \\
V(u(\tau), \tau) & =V(c(\tau), \tau)+K .
\end{aligned}
$$

\subsubsection{The information decision}

Firms always have the option to incur the information cost $F$ to gather and process information about the unobserved $W_{a, t}$ process. Upon doing so, they learn the realization of $W_{a, t}$ - or, equivalently, their frictionless optimal price $p_{t}^{*}$ - and the time elapsed since the last information date $\tau$ is reset to zero. The decision on whether or not to get informed at any given point $(z, \tau)$ involves comparing the value function at that point with the expected value function given the conditional distribution of the discrepancy that will be observed upon incurring the information cost. This implies that the value function must satisfy the following condition:

$$
\forall(z, \tau), \quad V(z, \tau) \leq E\left[V\left(z+\sigma_{a} \sqrt{\tau} \varepsilon, 0\right)\right]+F,
$$

where $\varepsilon$ is a standard normal random variable.

Points in the state space in which the firm is indifferent between getting informed and continuing with outdated information, for which (12) holds with equality, define the information boundary $\left(z, \tau^{*}(z)\right)$. Thus, on information dates the expected discrepancy receives a shock with distribution $N\left(0, \sigma_{a}^{2} \tau^{*}(z)\right)$, and $\tau$ is reset to zero, yielding the following informational matching condition:

$$
V\left(z, \tau^{*}(z)\right)=E\left[V\left(z+\sigma_{a} \sqrt{\tau^{*}(z)} \varepsilon, 0\right)\right]+F
$$




\subsubsection{The optimal rule}

Figure 1 illustrates the optimal pricing rule under adjustment and information costs, and partial information. ${ }^{15}$ The numerical methodology used for solving this problem, based on a finite difference approach can be found in Bonomo, Carvalho and Garcia (2011). When we jointly take into account the adjustment and information inaction regions, we do not need to consider information decisions at $(z, \tau)$ points that are outside the adjustment inaction region, since those points will never be reached. Likewise, there is no need to contemplate $(z, \tau)$ points that are outside the information inaction region. Thus, the resulting inaction region (depicted in Figure 1), in which no adjustment or information gathering occurs, is the intersection between the adjusment and information inaction regions.

Under the optimal rule, the firm uses $W_{i, t}$-information between information dates and adjusts the expected price discrepancy to $c(\tau)$ whenever it hits the $l(\tau)$ or $u(\tau)$ boundaries of the inaction region (outer dashed (red) lines, inherited from the adjustment inaction region). The inner dashed (red) line, $c(\tau)$, gives the discrepancy to which the firm reverts whenever it chooses to adjust. The solid (blue) line $\tau^{*}(z)$ is the boundary of the inaction region inherited from the information inaction region, which triggers information gathering.

Notice that the sizes of price adjustments implied by the optimal rule are not constant, as they would be under full information. ${ }^{16}$ Partially informed upward adjustments have size $c(\tau)-l(\tau)$, while partially informed price decreases have size $u(\tau)-c(\tau)$. Those adjustment sizes depend on the time elapsed since the last information date. In the example presented in Figure 1, in which there is a positive drift in the frictionless optimal price process, the partially informed upward adjustment size clearly increases with $\tau$. Fully informed adjustments are potentially much more variable in size, with lower bound $c(0)-l(0)$ for price increases and $u(0)-c(0)$ for price decreases.

In the sample path realization for the expected price discrepancy $\left(z_{t}\right)$ that we depict in Figure 1 (solid (black) line), there are two partially-informed adjustments before the firm decides to incur the cost to entertain information about $W_{a, t}$. At that point the time-elapsed variable $\tau$ is reset to zero, and the firm learns the cumulative innovation $W_{a, t}-W_{a, t-\tau^{*}(z)} \sim N\left(0, \sigma_{a}^{2} \tau^{*}(z)\right)$. Then, the firm decides whether or not to incur the menu cost and change its price, depending on whether the price discrepancy is inside or outside the inaction region defined by $(l(0), u(0))$.

For small $\tau$, the limits of the inaction region are dictated by the boundaries of the adjustment

\footnotetext{
${ }^{15}$ The parameter values used in this section are merely illustrative. In Section XX we estimate them using price setting statistics from micro data.

${ }^{16}$ In a pure menu-cost version of the model, our assumed process for the frictionless optimal price would generate constant adjustment sizes for both price increases and decreases. Of course adding mean reversion in the frictionless optimal price process, as in Golosov and Lucas (2008), or economics of scope in menu costs, as in Midrigan (2011), would add some size variability.
} 
inaction, whereas for large $\tau$ they are defined by the boundary of the information inaction region. When information about $W_{a, t}$ is not yet too stale, if partial information gives rise to a large enough expected price discrepancy, the firm finds it optimal to make a partially-informed adjustment. After some point (corresponding to $\tau \approx 0.65$ in Figure 1 ), making partially-informed adjustments is no longer optimal. The reason is that by that time the firm's information set has "depreciated" enough (due to the accumulation of unobserved innovations to $W_{a, t}$ ). Thus, a given expected discrepancy that might have triggered a partially-informed adjustment early on, will now trigger information gathering instead.

An interesting implication of optimal pricing behavior under adjustment and information costs, which can be glimpsed from the previous description, is that it is never optimal to make a partiallyinformed adjustment just prior to an information date. Rather than incurring the menu cost to make such an adjustment and then immediately incurring the information cost, it is always better to reverse the order of these actions and keep the option to adjust, to be exercized or not depending on the new information.

The principle that it is never optimal to make an adjustment just prior to the arrival of relevant information is rather general. The next section provides a context in which this principle manifests itself in a particularly stark fashion.

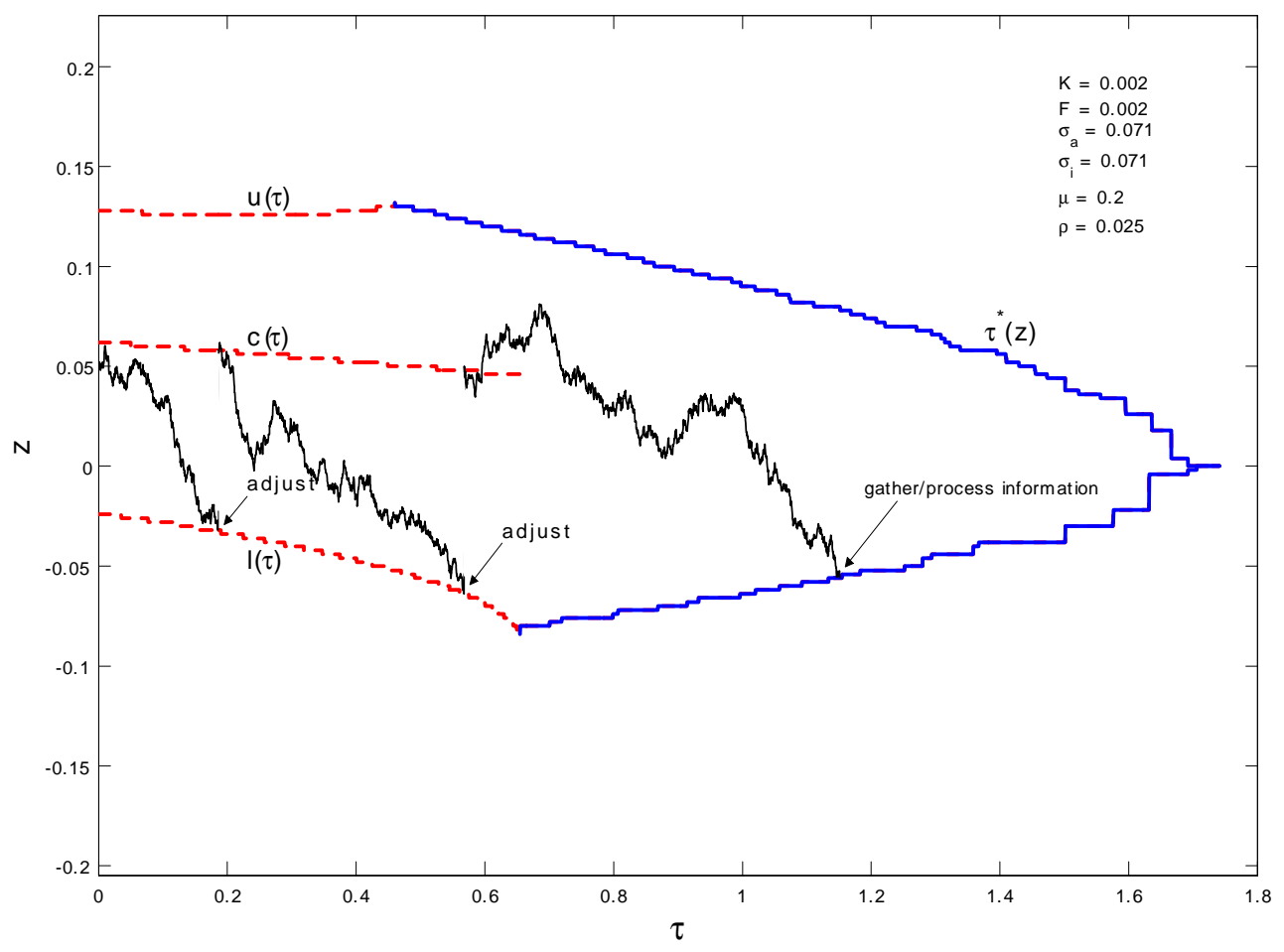


Optimal pricing policy with partial information, and adjustment and information costs

\subsection{Extreme inertia before information dates}

In this section we assume that information about $W_{a, t}$ arrives infrequently for reasons that are outside the control of firms. ${ }^{17}$ This case is analytically simpler than the problem with costly information gathering and processing, analyzed above. Nevertheless, it highlights in a striking way the importance of cumulative unobserved information for price adjustment decisions.

The function $f$ and the dynamics for $z$ are still given by (7) and (6), respectively. Therefore the differential form of the Bellman equation is also given by (8).

The condition that determines $c(\tau)$, (9), the adjustment-option condition, (10), and the conditions that determine $l(\tau)$ and $u(\tau),(11)$, remain the same. However, the value-matching condition now applies to the exogenous time elapsed since the prior information date, denoted $T$ :

$$
V(z, T)=E\left[V\left(z+\sigma_{a} \sqrt{T} \varepsilon, 0\right)\right]
$$

The numerical solution algorithm used to solve this problem, described in Appendix E, is similar to the one used in the costly information problem.

Figure 2 shows the optimal pricing rule and a sample path for $z_{t}$, assuming $T=1$. For $\tau$ between zero and one, adjustment is dictated by the evolution of the expected discrepancy, which depends on $\mu$ and on realizations of $W_{i, t}$. When $z_{t}$ reaches the lower barrier $l(\tau)$, adjustment is triggered to $c(\tau)$. These adjustments take into consideration only the continuously and freely available $W_{i, t}$-information. When $\tau$ reaches time $T=1, W_{a, t}$-information arrives and $z_{t}$ jumps. If it falls outside the inaction region for $\tau=0$, the firm changes its price, resetting the price discrepancy to $c(0)$.

In this environment there are no totally-uninformed adjustments. The firm uses the $W_{i, t^{-}}$ information between information dates, and adjusts if the expected price discrepancy becomes large enough. Despite the continuous flow of $W_{i, t}$-information, the inaction region becomes arbitrarily wide before the deterministic times of $W_{a, t}$-information arrival, clearly showing that, as in the case with costly information, it is never optimal to adjust immediately before becoming informed. In the case of exogenous deterministic information, since getting information is not an option, this principle has a slightly different meaning, implying that one should not adjust before an important information announcement.

\footnotetext{
${ }^{17}$ Examples of exogenously infrequent information are pervasive. Economic developments often become news after having evolved unnoticed for some time. Data releases on prespecified dates usually reflect cumulative past information about the state of the economy.
} 
In the case of prespecified information dates the inaction range becomes arbitrarily large just before information releases. The intuition is clear: by delaying adjustment the firm incurs additional flow costs due to the expected price discrepancy, but retains the option to adjust or not after it receives information. If the firm chooses not to adjust, it saves the lump-sum menu cost, which is infinitely larger than the discrepancy flow costs (over an infinitesimal time interval). Since there is a positive probability of not adjusting, just prior to the information date the option value of waiting becomes arbitrarily large relative to those flow costs. This is a stark testable implication of this specification: one should see fewer adjustments when potentially important information is about to be released.

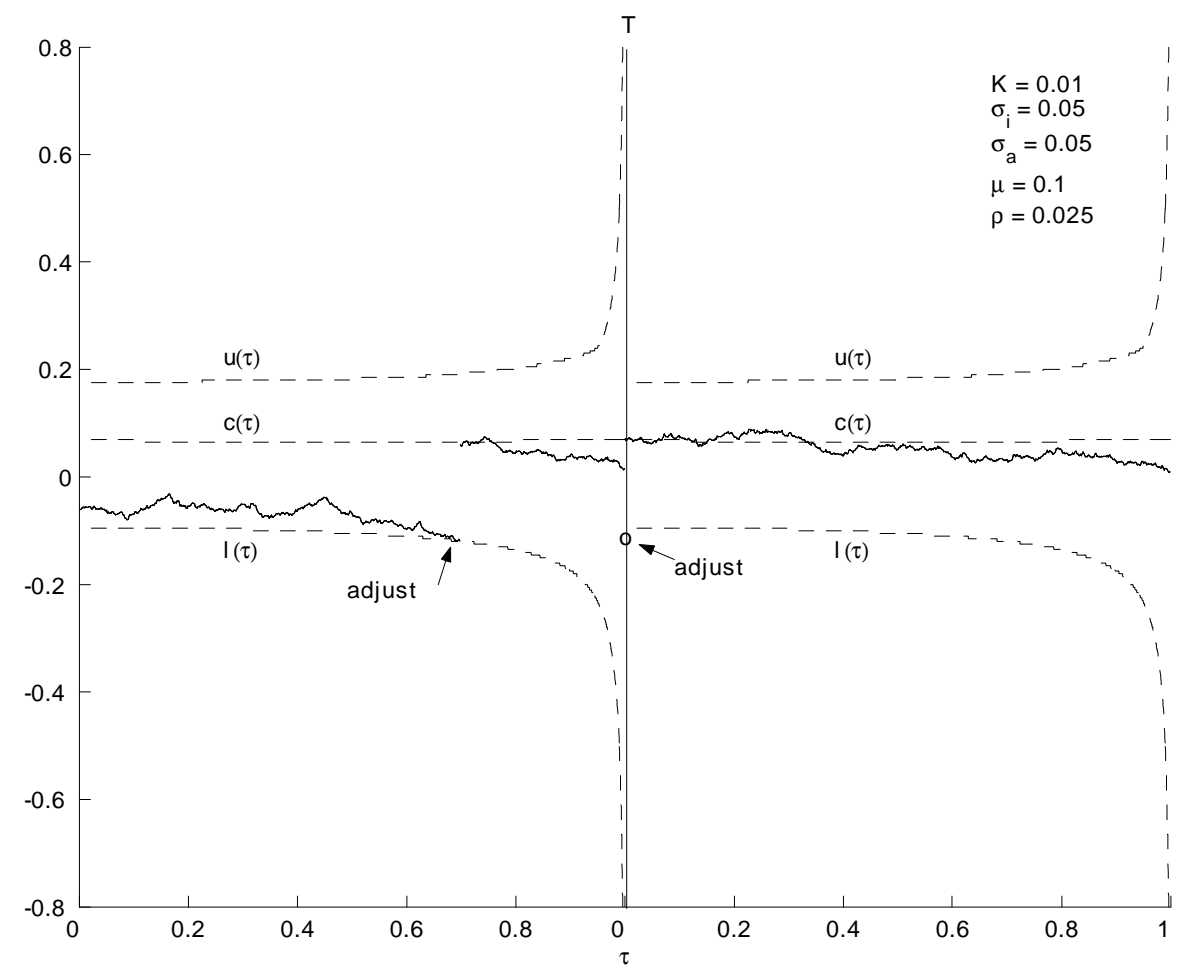

Optimal pricing policy under partial continuous information, and exogenous and deterministically infrequent information

\section{A special case with costly information only}

In this section, we use our framework to tackle a particular case of the problem in section 3 , where no information is freely observable. While in the setting of that section the model generated both partially informed and fully informed adjustments, now besides the fully informed adjustments there is the possility of having uninformed adjusments. If adjustment costs are relatively small with respect to information costs and inflation has a drift, the firm has an incentive to counteract 
the trend in the frictionless optimal price by adjusting more often than becoming informed.

Although this same problem of optimal adjustment and information acquisition has been posed by Alvarez, Lippi, and Paciello (2011), they preclude the possibility of uninformed adjustments since their framework did not keep track of the expected price discrepancy at dates different from information dates.

We solve this problem by setting $\sigma_{i}=0$ and relabeling $\sigma=\sigma_{a}$ in the setting of section 3 . Thus, the differential equation which characterizes the evolution of the value function inside the inaction range becames:

$$
-\mu V_{z}(z, \tau)+V_{\tau}(z, \tau)-\rho V(z, \tau)+z^{2}+\sigma^{2} \tau=0,
$$

with general solution given by (??):

$$
V(z, \tau)=\frac{2 \mu^{2}}{\rho^{3}}-\frac{2 z \mu}{\rho^{2}}+\frac{z^{2}}{\rho}+\frac{\sigma^{2}}{\rho^{2}}+\frac{\sigma^{2} \tau}{\rho}+e^{-\frac{\rho z}{\mu}} G\left(\frac{z+\mu \tau}{\mu}\right),
$$

where $G(\cdot)$ is a function to be determined by the nature of the firm's optimization problem.

The conditions related to the adjustment decision (9, 10 and 11) and information decision $(12,13)$ remain the same.

In Appendix E we provide an algorithm for solving this problem numerically using a finitedifference method. Figure 4 illustrates the optimal pricing rule under adjustment and information gathering/processing costs. The dashed (red) lines $l(\tau), u(\tau)$ are the boundaries of the inaction region that trigger uninformed adjustments, while the solid (blue) line $\tau^{*}(z)$ is the boundary that triggers information gathering/processing. We illustrate a sample path in which $z_{t}$ is initially close to zero. Due to the high enough drift $\mu$, the expected discrepancy hits the lower boundary $l(\tau)$, leading to an uninformed adjustment to $c(\tau)$. After that, the expected discrepancy drifts down until it touches the information boundary $\tau^{*}(z)$ at a point where $z \approx-0.04$ and $\tau \approx 0.73$. At that point the firm incorporates information into the pricing decision, as the expected discrepancy receives a shock with distribution $N\left(0, \sigma^{2} \times 0.73\right)$. The time-elapsed variable $\tau$ is reset to zero, and the firm decides whether or not to pay the menu cost and change its price, depending on whether the just-learned price discrepancy is inside or outside the information-date inaction region, defined by $(l(0), u(0))$.

As in the case with deterministic information releases, notice that it is never optimal to make an uninformed price adjustment just prior to an information date. This result can be seen visually in the example depicted in Figure 4. For $\tau>0$ the (red) dashed $l(\tau)$ and $u(\tau)$ lines trigger adjustment without information. Such an adjustment brings the expected discrepancy to $c(\tau)$, which is always "distant" from the information boundary $\tau^{*}(z)$ that triggers information gathering/processing. The 
intuition for this feature of the optimal policy is similar to the case with deterministic information releases, the difference arising from the fact that in the current problem information is controlled by the price setter: rather than incurring the menu cost to make an uninformed adjustment and then immediately incurring the information gathering/processing cost, it is always better to reverse the order of these actions and keep the option to adjust, to be exercized or not depending on the new information.

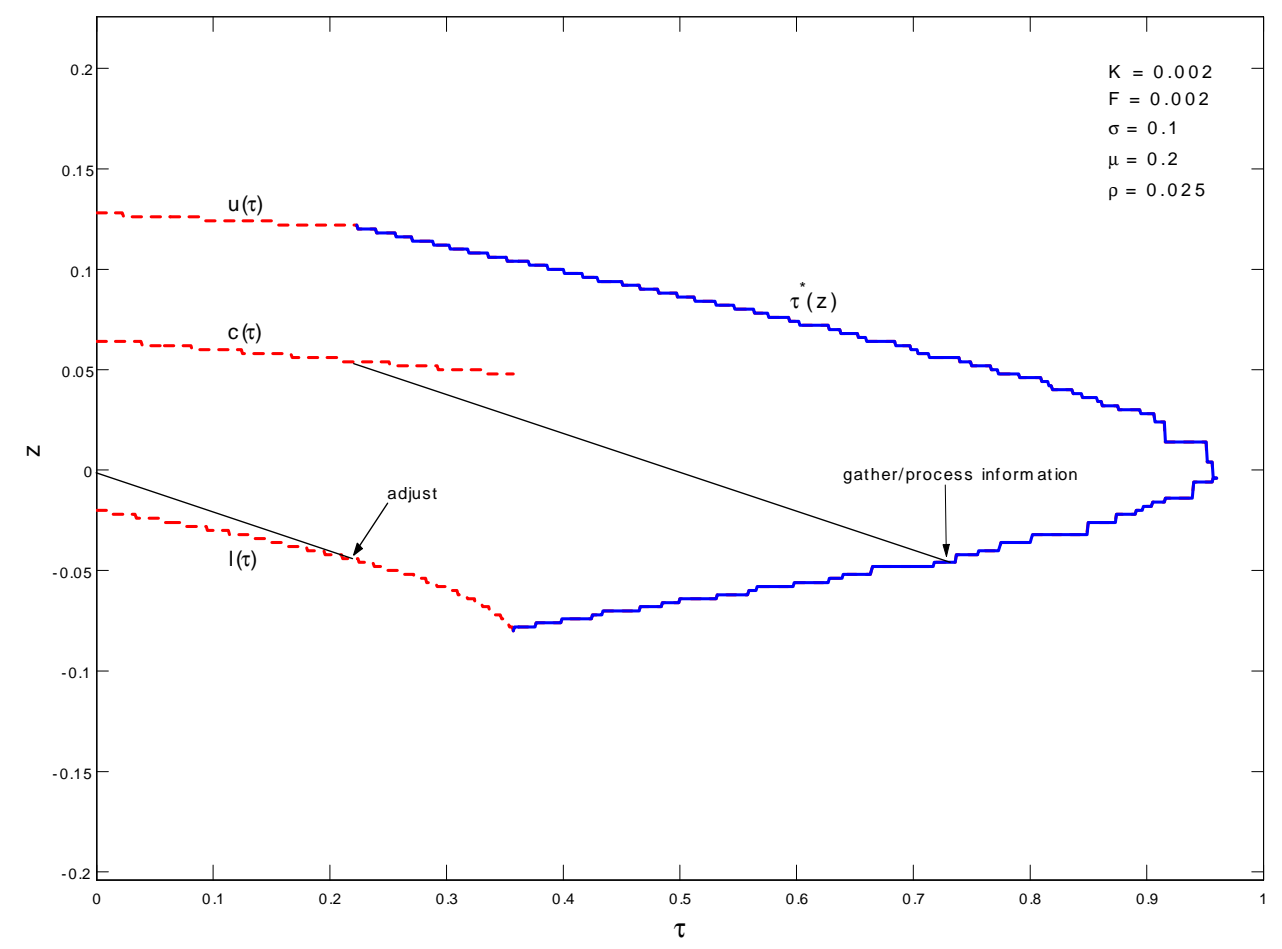

Figure 1: Optimal pricing policy under adjustment and information gathering/processing costs

Alvarez, Lippi, and Paciello (2010) also propose a price-setting model with costly adjustments and information acquisition. Beyond the difference in the solution approach, another key difference is that our approach to solving the problem with dissociated costs entertains cases in which uninformed adjustments are optimal. In turn, Alvarez, Lippi and Paciello (2010) provide sufficient conditions under which such uninformed adjustments are not optimal and focus on parameterizations that satisfy those conditions. They otherwise prevent agents from making such adjustments by imposing the restriction that adjustment requires observation. ${ }^{18}$

In this example we assume the same parameter values as in Subsection ??, splitting the sources

\footnotetext{
${ }^{18}$ For a more detailed discussion of the differences between our papers see Bonomo, Carvalho, and Garcia (2011).
} 
of variation of the $p_{t}^{*}$ process evenly between the $W_{i, t}$ and $W_{a, t}$ processes (i.e. $\sigma_{i}=\sigma_{a}=\sigma / \sqrt{2}$ ). This leads to a quite dramatic change in the optimal pricing policy, in that the firm is now willing to wait longer until the subsequent information date than in the case without interim information. This is quite intuitive, since the expected flow deviation cost due to unobserved variation in $p_{t}^{*}$ is now smaller.

\section{Conclusion}

In this paper we study optimal price setting under adjustment costs and infrequent information arising from various sources. Pricing rules are more complex than the usual purely state-dependent strategies. In general, the inaction regions depend on the time elapsed since the last information date. There is scope for uninformed adjustments. When some important determinant of the frictionless optimal price can be freely and continuously factored into pricing decisions, there can be partially-informed adjustments. There should be no adjustment just prior to the release of important information, if the release date is known. Likewise, it is never optimal to make an uninformed price adjustment just before incurring the information gathering and processing cost.

While in this paper we focus on price setting, as emphasized in the early Bonomo and Garcia (2003) paper, our framework is, more generally, suitable for studying optimal decision-making under adjustment costs and infrequent information. For instance, our results might be of interest in the context of employment adjustment, inventory management, and investment problems.

In its current form, our framework has the big advantage that the optimal policies can be solved for independently of equilibrium considerations. This makes the various models that we entertain relatively cheap to solve computationally, and thus allows for a relatively straightforward attack on their quantitative micro and macro implications. Of course such simplicity, which is afforded by the nature of the underlying economic environment, also has some costs. Importantly, it precludes interactions of agents' decisions through general-equilibrium effects. While our formulations can be extended to allow for such interations, they have to be handled with methods for solving (infinitely-dimensional) heterogeneous agents models, which typically make computational solutions more costly. Despite this complication, solutions are feasible, and should open the possibility of addressing important research questions. 


\section{References}

[1] Abel, A., J. Eberly and S. Panageas (2009), "Optimal Inattention to the Stock Market with Information Costs and Transactions Costs," NBER Working Paper \#15010.

[2] Almeida, H. and M. Bonomo (2002), "Optimal State-Dependent Rules, Credibility and Inflation Inertia," Journal of Monetary Economics 49: 1317-1336.

[3] Alvarez, F., L. Guiso, and F. Lippi (2010), "Durable Consumption and Asset Management with Transaction and Observation Costs," NBER Working Paper \#15835.

[4] Alvarez, F., F. Lippi, and L. Paciello (2010), "Optimal Price Setting with Observation and Menu Costs," NBER Working Paper \#15852.

[5] Ball, L., G. Mankiw and D. Romer (1988), "The New Keynesian Economics and the OutputInflation Trade-off," Brookings Papers on Economic Activity 1: 1-65.

[6] Ball, L. and D. Romer (1990), "Real Rigidities and the Non-Neutrality of Money," Review of Economic Studies 57: 183-203.

[7] Bils, M. and P. Klenow (2004), "Some Evidence on the Importance of Sticky Prices," Journal of Political Economy 112: 947-985.

[8] Bonomo, M. and C. Carvalho (2004), "Endogenous Time-Dependent Rules and Inflation Inertia," Journal of Money, Credit and Banking 36: 1015-1041.

[9] (2010), "Imperfectly-Credible Disinflation under Endogenous Time-Dependent Pricing," Journal of Money, Credit and Banking 42: 799-831.

[10] Bonomo, M., C. Carvalho, and R. Garcia (2011), "State-dependent Pricing under Infrequent Information: A Unified Framework," Federal Reserve Bank of New York Staff Reports \#455.

[11] Bonomo, M. and R. Garcia (1994), "Indexation, Staggering and Disinflation," Journal of Development Economics 43: 39-58.

[12] (2001), "The Macroeconomic Effects of Infrequent Information with Adjustment Costs," Canadian Journal of Economics 34: 18-35.

$[13]$ (2003), "Optimal Rules under Adjustment Cost and Infrequent Information," mimeo available at http://repec.org/esLATM04/up.7597.1081885890.pdf. 
[14] Burstein, A. (2006), "Inflation and Output Dynamics with State-Dependent Pricing Decisions," Journal of Monetary Economics 53: 1235-1257.

[15] Caballero, R. (1989), "Time Dependent Rules, Aggregate Stickiness and Information Externalities," Working Paper no. 428, Columbia University.

[16] Calvo, G. (1983), "Staggered Prices in a Utility Maximizing Framework," Journal of Monetary Economics 12: 383-98.

[17] Caplin, A. and J. Leahy (1991), "State-Dependent Pricing and the Dynamics of Money and Output," Quarterly Journal of Economics 106: 683-708.

[18] _ (1997), "Aggregation and Optimization with State-Dependent Pricing," Econometrica 65: 601-625.

[19] Caplin, A. and D. Spulber (1987), "Menu Costs and the Neutrality of Money," Quarterly Journal of Economics 102: 703-726.

[20] Danziger, L. (1999), “A Dynamic Economy with Costly Price Adjustments," American Economic Review 89: 878-901.

[21] Dhyne, E., L. Álvarez, H. Le Bihan, G. Veronese, D. Dias, J. Hoffman, N. Jonker, P. Lünnemann, F. Rumler and J. Vilmunen (2006), "Price Changes in the Euro Area and the United States: Some Facts from Individual Consumer Price Data," Journal of Economic Perspectives 20: $171-192$.

[22] Dias, D., C. Marques and J. Silva (2007), "Time- or state-dependent price setting rules? Evidence from micro data," European Economic Review 51: 1589-1613.

[23] Gertler, M. and J. Leahy (2008), "A Phillips Curve with an Ss Foundation," Journal of Political Economy 116: 533-572.

[24] Golosov, M. and R. Lucas (2007), "Menu Costs and Phillips Curves," Journal of Political Economy 115: 171-199.

[25] Gorodnichenko, Y. (2008), "Endogenous Information, Menu Costs and Inflation Persistence," NBER Working Paper No. 14184.

[26] Jadresic, E. (2002), "The Macroeconomic Consequences of Wage Indexation Revisited," in F. Lefort, and K. Schmidt-Hebbel (orgs.), Indexation, Inflation and Monetary Policy, Central Bank of Chile. 
[27] Klenow, P., and O. Kryvtsov (2008), "State-Dependent or Time-Dependent Pricing: Does It Matter for Recent U.S. Inflation?" Quarterly Journal of Economics 123: 863-904

[28] Klenow, P. and J. Willis (2007), "Sticky Information and Sticky Prices," Journal of Monetary Economics 54: 79-99.

[29] Knotek, E. (2010), "A Tale of Two Rigidities: Sticky Prices in a Sticky-Information Environment," Journal of Money, Credit, and Banking 42: 1543-64.

[30] Kongsamut, P., S. Rebelo and D. Xie (2001), "Beyond Balanced Growth," Review of Economic Studies 68: 869-882.

[31] Kryvtsov, O. (2009), "Information Flows and Aggregate Persistence," mimeo available at http://www.kryvtsov.com.

[32] Maćkowiak, B., and M. Wiederholt (2008), "Optimal Sticky Prices under Rational Inattention," American Economic Review 99: 769-803.

[33] Mankiw, G., and R. Reis (2002), "Sticky Information Versus Sticky Prices: A Proposal to Replace the New Keynesian Phillips Curve," Quarterly Journal of Economics 117: 1295-1328.

[34] Midrigan, V. (2010), "Is Firm Pricing State or Time-Dependent? Evidence from US Manufacturing," Review of Economics and Statistics 92: 643-656.

[35] _ (2011), "Menu Costs, Multi-Product Firms and Aggregate Fluctuations," Econometrica 79: 1139-1180.

[36] Moscarini, G. (2004), "Limited Information Capacity as a Source of Inertia," Journal of Economic Dynamics and Control 28: 2003-2035.

[37] Nakamura, E. and J. Steinsson (2010), "Monetary Non-Neutrality in a Multi-Sector Menu Cost Model," Quarterly Journal of Economics 125: 961-1013.

[38] Phelps, E. (1978), "Disinflation without Recession: Adaptive Cuideposts and Monetary Policy," Weltwirtschaftliches Archiv 114: 783-809.

[39] Reis, R. (2006), "Inattentive Producers," Review of Economic Studies 73: 793-821.

[40] Sheshinski, E. and Y. Weiss (1977), "Inflation and Costs of Price Adjustment," Review of Economic Studies 44: 287-304. 
[41] Taylor, J. (1979), "Staggered Price Setting in a Macro Model," Journal of Political Economy 88: 1-24.

[42] Taylor, J. (1980), "Aggregate Dynamics and Staggered Contracts," Journal of Political Economy 88: 1-23.

[43] Woodford, M. (2003), Interest and Prices: Foundations of a Theory of Monetary Policy, Princeton University Press.

[44] _ (2009), "Information-Constrained State-Dependent Pricing," Journal of Monetary Economics 56, Supplement: S100-S124.

[45] Yun, T. (1996), "Nominal Price Rigidity, Money Supply Endogeneity, and Business Cycles," Journal of Monetary Economics 37: 345 - 370. 


\section{Appendix A}

We derive the frictionless optimal price in a simple general equilibrium framework. A representative consumer maximizes expected discounted utility:

$$
E_{t_{0}} \int_{t_{0}}^{\infty} e^{-\rho\left(t-t_{0}\right)}\left[\log \left(C_{t}\right)-H_{t}\right] d t
$$

subject to the budget constraints:

$$
B_{t}=B_{0}+\int_{0}^{t} W_{r} H_{r} d r-\int_{0}^{t}\left(\int_{0}^{1} P_{i r} C_{i r} d i\right) d r+\int_{0}^{t} T_{r} d r+\int_{0}^{t} \Lambda_{r} d Q_{r}+\int_{0}^{t} \Lambda_{r} d D_{r}, \text { for } t \geq 0 .
$$

Utility is defined over the composite consumption good $C_{t} \equiv\left[\int_{0}^{1}\left(C_{i t} / A_{i t}\right)^{\frac{\theta-1}{\theta}} d i\right]^{\frac{\theta}{\theta-1}}$ with $\theta>1$, where $C_{i t}$ is the consumption of variety $i$, and $A_{i t}$ is a relative-preference shock. $P_{i t}$ is the price of variety $i, H_{t}$ is the supply of labor, which commands a wage $W_{t}, B_{t}$ is total financial wealth, $T_{t}$ are total net transfers, including any lump-sum flow transfer from the government, and profits received from the firms owned by the representative consumer. $Q_{r}$ is the vector of prices of traded assets, $D_{r}$ is the corresponding vector of cumulative dividend processes, and $\Lambda_{r}$ is the trading strategy, which satisfies conditions that preclude Ponzi schemes. The associated consumption price index, $P_{t}$, is given by:

$$
P_{t}=\left[\int_{0}^{1} P_{i t}^{1-\theta} d i\right]^{\frac{1}{1-\theta}}
$$

The demand for an individual variety is:

$$
C_{i t}=A_{i t}^{1-\theta}\left(\frac{P_{i t}}{P_{t}}\right)^{-\theta} C_{t}
$$

Firms hire labor to produce according to the following production function:

$$
Y_{i t}=A_{i t} H_{i t}
$$

Note that we assume that the productivity shock is perfectly correlated with the relative-preference shock in the consumption aggregator. This has precedence in the sticky-price literature (for instance, King and Wolman 1999 and Woodford 2009). Our specific assumption follows Woodford (2009), and aims to produce a tractable profit-maximization problem that can be written as a price-setting "tracking problem" in which the firm only cares about the ratio of the two stochastic processes driving profits, which will be specified below. ${ }^{19}$

\footnotetext{
${ }^{19}$ More generally, assumptions relating preference and technology processes have been used previously in the literature on "balanced growth" in multi-sector models (e.g. Kongsamut et al. 2001).
} 
The static profit-maximizing price for firm $i, P_{i t}^{*}$ (also referred to as its frictionless optimal price), is given by the usual markup rule:

$$
P_{i t}^{*}=\frac{\theta}{\theta-1} \frac{W_{t}}{A_{i t}}
$$

From the representative household's labor supply:

$$
\frac{W_{t}}{P_{t}}=C_{t}
$$

which leads to:

$$
P_{i t}^{*}=\frac{\theta}{\theta-1} \frac{P_{t} C_{t}}{A_{i t}} .
$$

In logarithms (lowercase variables denote logarithms throughout), this reads:

$$
p_{i t}^{*}=\log \left(\frac{\theta}{\theta-1}\right)+\log \left(P_{t} C_{t}\right)-\log \left(A_{i t}\right) .
$$

Ignoring the unimportant constant and assuming appropriate exogenous stochastic processes for nominal aggregate demand and for idiosyncratic productivity yields the specifications used throughout the main text.

\section{Appendix B}

Here we derive the quadratic approximation to the static profit-maximization problem used in the main text. Write real flow profits as:

$$
\Pi\left(\frac{P_{i}}{P}, C, A_{i}\right)=A_{i}^{1-\theta} \frac{P_{i}}{P}\left(\frac{P_{i}}{P}\right)^{-\theta} C-\frac{W}{P A_{i}} A_{i}^{1-\theta}\left(\frac{P_{i}}{P}\right)^{-\theta} C,
$$

where $P_{i}$ is the price charged by firm $i$. We can use the labor supply equation to express the real wage as a function of aggregate consumption $\left(\frac{W}{P}=C\right)$, and rewrite the expression for real flow profits as:

$$
\Pi\left(\frac{P_{i}}{P}, C, A_{i}\right)=A_{i}^{1-\theta}\left(\frac{P_{i}}{P}\right)^{1-\theta} C-C^{2} A_{i}^{-\theta}\left(\frac{P_{i}}{P}\right)^{-\theta} .
$$

Let $\bar{\Pi}$ be the steady-state level of real profits in a frictionless economy (upper bars denote steady-state values): ${ }^{20}$

$$
\bar{\Pi} \equiv \Pi\left(\frac{P_{i}^{*}}{P}, \bar{C}, A_{i}\right)
$$

\footnotetext{
${ }^{20} \mathrm{~A}$ constant level of aggregate consumption requires the restriction $\left[\int_{0}^{1} A_{i t}^{\theta-1} d i\right]^{\frac{1}{1-\theta}}=1$, which we assume holds throughout the paper.
} 
We want to approximate the loss function $\overline{\bar{L}}$ defined as:

$$
\begin{aligned}
\overline{\bar{L}}\left(\frac{P_{i}^{*}}{P}, \frac{P_{i}}{P}, C, A_{i}\right) & =\frac{\Pi\left(\frac{P_{i}^{*}}{P}, C, A_{i}\right)-\Pi\left(\frac{P_{i}}{P}, C, A_{i}\right)}{\bar{\Pi}} \\
& =\frac{\Pi\left(\frac{P_{i}^{*}}{P}, C, A_{i}\right)-\Pi\left(\frac{P_{i}}{P}, C, A_{i}\right)}{\Pi\left(\frac{P_{i}^{*}}{P}, C, A_{i}\right)} \frac{\left.P_{i}^{*}, \bar{C}, A_{i}\right)}{\bar{\Pi}},
\end{aligned}
$$

The second ratio in (17) can be written as:

$$
\begin{aligned}
\frac{\Pi\left(\frac{P_{i}^{*}}{P}, C, A_{i}\right)}{\bar{\Pi}} & =\frac{A_{i}^{1-\theta}\left(\frac{P_{i}^{*}}{P}\right)^{1-\theta} C-C^{2} A_{i}^{-\theta}\left(\frac{P_{i}^{*}}{P}\right)^{-\theta}}{\bar{C}-\bar{C}^{2}} \\
& =\frac{A_{i}^{1-\theta}\left(\frac{P_{i}^{*}}{P}\right)^{1-\theta} C-\frac{\theta-1}{\theta} C A_{i}^{1-\theta}\left(\frac{P_{i}^{*}}{P}\right)^{1-\theta}}{\bar{C}-\frac{\theta-1}{\theta} \bar{C}} \\
& =A_{i}^{1-\theta} \frac{C}{\bar{C}}\left(\frac{P_{i}^{*}}{P}\right)^{1-\theta} \\
& =\left(\frac{C}{\bar{C}}\right)^{2-\theta},
\end{aligned}
$$

where we use the facts that $\frac{P_{i}^{*}}{P}=\frac{\theta}{\theta-1} \frac{C}{A_{i}}$ and $\bar{C}=\frac{\theta-1}{\theta}$. Note how the link between preferences and technology makes the idiosyncratic shock drop from the expression for maximized profits.

The first ratio in (17) is the proportional profit loss due to the "suboptimal" price $P_{i}$. It is convenient to rewrite it as:

$$
\frac{\Pi\left(\frac{P_{i}^{*}}{P}, C, A_{i}\right)-\Pi\left(\frac{P_{i}}{P}, C, A_{i}\right)}{\Pi\left(\frac{P_{i}^{*}}{P}, C, A_{i}\right)}=1-\frac{\Pi\left(\frac{P_{i}}{P}, C, A_{i}\right)}{\Pi\left(\frac{P_{i}^{*}}{P}, C, A_{i}\right)} .
$$

The profit ratio in the above expression can be written as:

$$
\begin{aligned}
\frac{\Pi\left(\frac{P_{i}}{P}, C, A_{i}\right)}{\Pi\left(\frac{P_{i}^{*}}{P}, C, A_{i}\right)} & =\frac{A_{i}^{1-\theta}\left(\frac{P_{i}}{P}\right)^{1-\theta} C-C^{2} A_{i}^{-\theta}\left(\frac{P_{i}}{P}\right)^{-\theta}}{A_{i}^{1-\theta}\left(\frac{P_{i}^{*}}{P}\right)^{1-\theta} C-C^{2} A_{i}^{-\theta}\left(\frac{P_{i}^{*}}{P}\right)^{-\theta}} \\
& =\frac{A_{i}^{1-\theta}\left(\frac{P_{i}}{P}\right)^{1-\theta}-\frac{\theta-1}{\theta} \frac{P_{i}^{*}}{P} A_{i}^{1-\theta}\left(\frac{P_{i}}{P}\right)^{-\theta}}{A_{i}^{1-\theta}\left(\frac{P_{i}^{*}}{P}\right)^{1-\theta}-\frac{\theta-1}{\theta} \frac{P_{i}^{*}}{P} A_{i}^{1-\theta}\left(\frac{P_{i}^{*}}{P}\right)^{-\theta}} \\
& =\theta \frac{\left(\frac{P_{i}}{P}\right)^{1-\theta}-\frac{\theta-1}{\theta} \frac{P_{i}^{*}}{P}\left(\frac{P_{i}}{P}\right)^{-\theta}}{\left(\frac{P_{i}^{*}}{P}\right)^{1-\theta}} \\
& =\theta\left(\frac{P_{i}^{*}}{P_{i}}\right)^{\theta-1}-(\theta-1)\left(\frac{P_{i}^{*}}{P_{i}}\right)^{\theta},
\end{aligned}
$$


so that:

$$
\frac{\Pi\left(\frac{P_{i}^{*}}{P}, C, A_{i}\right)-\Pi\left(\frac{P_{i}}{P}, C, A_{i}\right)}{\Pi\left(\frac{P_{i}^{*}}{P}, C, A_{i}\right)}=1-\theta\left(\frac{P_{i}^{*}}{P_{i}}\right)^{\theta-1}+(\theta-1)\left(\frac{P_{i}^{*}}{P_{i}}\right)^{\theta} .
$$

As before, note how the link between preference and technology makes the idiosyncratic shock drop from the expression above.

Combining (18) and (19), and keeping the relevant arguments of the loss function, we obtain:

$$
\overline{\bar{L}}\left(\frac{P_{i}^{*}}{P}, \frac{P_{i}}{P}, C, A_{i}\right)=\bar{L}\left(\frac{P_{i}^{*}}{P_{i}}, C\right)=\left(\frac{C}{\bar{C}}\right)^{2-\theta}\left[1-\theta\left(\frac{P_{i}^{*}}{P_{i}}\right)^{\theta-1}+(\theta-1)\left(\frac{P_{i}^{*}}{P_{i}}\right)^{\theta}\right] .
$$

We can rewrite the loss function $\bar{L}$ in terms of logarithms:

$$
G\left(p_{i}^{*}-p_{i}, c\right)=e^{(2-\theta) c}\left[\left(1-\theta e^{(\theta-1)\left(p_{i}^{*}-p_{i}\right)}\right)+(\theta-1) e^{\theta\left(p_{i}^{*}-p_{i}\right)}\right] .
$$

The exact loss function $G\left(p_{i}^{*}-p_{i}, c\right)$ can be used in the optimal price-setting problems. However, the presence of aggregate consumption in the expression implies that solving for the optimal pricing rule in the presence of pricing frictions involves a fixed-point problem, even in the absence of strategic complementarity or substitutability in price setting. To make the optimal pricing problem more tractable, we eliminate the effect of aggregate output by assuming $\theta=2$ (as in Danziger 1999 and Bonomo and Carvalho 2010). In addition, for analytical convenience we take a second-order Taylor expansion of flow profit losses around the frictionless optimal price, based on which we analyze the price-setting problems discussed in the paper:

$$
\text { flow profit losses }\left(p_{i t}\right) \propto\left(p_{i t}-p_{i t}^{*}\right)^{2} \text {. }
$$

